\title{
Comparing the Potential for Irritation of a Ceramide- Based Moisturizer with a Urea-Based Moisturizer for Pediatric Atopic Dermatitis
}

\author{
Valerie Pui Yoong Ho (D) - Eric Ma · Hui Min Liew • Michelle Si Ying Ng • \\ Mark Jean Aan Koh
}

Received: April 1, 2020 / Published online: May 6, 2020

(C) The Author(s) 2020

\section{ABSTRACT}

Introduction: Moisturizers are one of the mainstays of the topical treatment of atopic dermatitis (AD). One of the adverse effects of moisturizers is skin irritation, especially on excoriated AD skin. We compared the potential for irritation of two commercially available moisturizer products for the treatment of AD: a ceramide-based moisturizer (Ceradan ${ }^{\circledR}$ Cream; Hyphens Pharma Pte Ltd, Singapore) and a urea 5\% moisturizer (Aqurea Lite Cream; ICA Pharma Pte Ltd, Singapore).

Methods: We performed a prospective singleblind randomized controlled study recruiting AD patients aged between 8 and 16 years with symmetrical or near symmetrical scratch marks (excoriations) of at least grade 2 to 3 severity score, according to the Eczema Area and

Digital Features To view digital features for this article go to https://doi.org/10.6084/m9.figshare.12200345.

V. P. Y. Ho $(\bowtie) \cdot$ E. Ma $\cdot$ H. M. Liew

M. S. Y. Ng • M. J. A. Koh

Dermatology Service, KK Women's and Children's

Hospital, Singapore, Singapore

e-mail: valerie.ho.p.y@singhealth.com.sg
Severity Index (EASI), over bilateral antecubital fossae. Subjects were randomized to receive the ceramide-based moisturizer to either the left or right antecubital fossa or urea $5 \%$ cream to the other antecubital fossa. Subjects were asked to grade the immediate skin irritation of both creams on a standard visual analogue scale (VAS) and which cream they would prefer to use as a daily moisturizer. Primary outcome was the mean irritant score of each cream, and secondary outcome was the subjects' preference of either cream as their daily moisturizer.

Results: A total of 42 participants were enrolled with a mean age of 11 years 5 months. The ceramide-based cream had a significantly lower mean VAS score (mean $0.69, \mathrm{SD}=1.63$ ) for irritation compared with urea $5 \%$ cream $(1.43$, $\mathrm{SD}=1.64)(p=0.035)$. More participants also preferred the ceramide-based cream over urea $5 \%$ cream (62\% versus $38 \%)$ as their daily moisturizer, but this did not reach statistical significance $(p=0.164)$.

Conclusions: A ceramide-based moisturizer may be considered as a suitable choice for children to minimize irritation from moisturizer treatment for AD.

Keywords: Atopic dermatitis; Ceramide; Eczema; Emollient; Moisturizer; Skin irritation; Skin irritancy; Urea 


\section{Key Summary Points}

\section{Why carry out this study?}

AD is common and affects up to $20 \%$ of children worldwide.

Moisturizers are one of the mainstays of the topical treatment of $\mathrm{AD}$, and skin irritation is one of its common adverse effects.

Paucity of literature exists for direct comparison between moisturizers and their potential for skin irritation in the pediatric population.

We compared the potential for irritation of two commercially available skin moisturizer products for the treatment of $\mathrm{AD}$ : a ceramide-based cream versus a urea $5 \%$ cream.

\section{What was learned from the study?}

The ceramide-based moisturizer had a significantly lower mean VAS score (mean $0.69, \mathrm{SD}=1.63)$ for irritation compared with urea $5 \%$ cream $(1.43, \mathrm{SD}=1.64)$, $p=0.035$.

More participants also preferred the ceramide-based cream over urea 5\% cream (62\% versus $38 \%$ ) as their daily moisturizer, but this did not reach statistical significance $(p=0.164)$

A ceramide-based moisturizer may be considered as a suitable choice for children to minimize irritation from moisturizer treatment of AD.

\section{INTRODUCTION}

Atopic dermatitis (AD) is a chronic inflammatory dermatosis characterized by recurrent, dry, irritated, and itchy skin. AD is common and affects up to $20 \%$ of children worldwide [1]. The skin of atopic individuals has been shown to be deficient in ceramides, filaggrin, and natural moisturizing factors, leading to increased transepidermal water loss and epidermal microfissuring [2].

Moisturizers help to improve barrier function, increase stratum corneum hydration in the chronically xerotic skin of AD individuals, and are essential in the treatment and prevention of $\mathrm{AD}$ [2]. There is great diversity with regards to the composition of moisturizers, which can be formulated to include varying proportions of humectants (e.g., urea or glycerol) that promote stratum corneum hydration, physiological lipids (e.g., ceramides, cholesterol, and fatty acids), which replenish and restore the intercellular lipid matrix of the stratum corneum, and occlusives (e.g., liquid paraffin and petrolatum), which work by creating a hydrophobic barrier over the skin and contribute to the efficacy of the intercellular lipid domains [3-6]. Side effects reported from the use of moisturizers include itching, burning, stinging, and redness [7]. These complications are more common on excoriated skin of $\mathrm{AD}$ patients. Considerations regarding the tolerability of moisturizers are important as this can impact compliance and may even lead to phobia of application, especially in children. By understanding the potential for irritation of moisturizers, we can recommend safe and effective moisturizer therapies to our patients and improve treatment compliance.

This study aims to compare the potential for irritation of two commercially available skin moisturizer products that have been developed for the treatment of $\mathrm{AD}$. We compared Ceradan ${ }^{\circledR}$ cream, a formulation containing three naturally found lipids, ceramides, cholesterol, and fatty acids, with Aqurea Lite cream, a readily available urea 5\% cream widely used in pediatric patients with $\mathrm{AD}$.

\section{METHODS}

\section{Study Design}

The present work is a prospective single-blind randomized controlled study. 


\section{Study Population}

Patients were recruited from the Pediatric Dermatology outpatient clinics at KK Women's and Children's Hospital (KKH), a tertiary pediatric hospital in Singapore, from January 2017 to March 2018.

Patients aged between 8 and 16 years diagnosed with $\mathrm{AD}$, according to the UK working party's criteria, with near symmetrical scratch marks (excoriations) of at least grade 2 to 3 severity score, according to the Eczema Area and Severity Index (EASI) over bilateral antecubital fossae, were included. Subjects must not have used that ceramide-based moisturizer or urea cream 3 months prior to recruitment. The test sites over the antecubital fossa should be free of scars, excessive hair, or tattoos that may interfere with objective measurements. Subjects had to be capable of understanding and following instructions.

Patients were excluded if they had used white soft paraffin or liquid paraffin moisturizers in the preceding 3 months, had concomitant skin disease other than $\mathrm{AD}$, were mentally incapable, had other significant medical conditions, or were on ongoing treatment with immunosuppressants, phototherapy, or oral or topical analgesics.

This study was approved by the SingHealth Centralised Institutional Review Board (CIRB), reference number 2015/3026, and conducted according to Good Clinical Practice guidelines and applicable local regulatory requirements. All procedures followed were in accordance with the ethical standards of the Helsinki Declaration of 1964, as revised in 2013. Written consent was obtained from parents or legal guardians and written assent was obtained from patients.

\section{Outcome Measures}

The primary outcome of the study was the mean irritant score of each cream. The secondary outcome was the subjects' preference of either of the two creams as their daily moisturizer.

\section{Randomization, Blinding, and Intervention}

After recruitment, subjects were randomized to receive the ceramide-based moisturizer to either the left or right antecubital fossa or urea 5\% cream to the other antecubital fossa. Randomization was computer generated. The creams were randomly labeled A and B, and the subjects were blinded to the type of cream applied on each antecubital fossa. The cream was always applied onto the subject's right antecubital fossa first, followed by the left. Each side was applied evenly with a flattened-out layer of a teaspoon quantity $(5 \mathrm{ml})$ of the respective cream.

Subjects were asked to grade the degree of immediate skin irritation after application of each cream on a standard visual analogue scale (VAS), with 1 being no irritation and 10 being extreme irritation. The degree of irritation was defined as stinging, burning, or pain. Scoring was performed within 5 min of application of the first cream over the right antecubital fossa. The other cream was applied to the left antecubital fossa $15 \mathrm{~min}$ later, and the irritation score was similarly performed within $5 \mathrm{~min}$ of application. At the end of the study, subjects were also asked which cream they would prefer to use as a daily moisturizer.

\section{Statistical Analysis}

Statistical analysis was performed using SPSS Statistics V25.0. Wilcoxon signed-rank test was used to compare the degree of irritation. Binomial test was used to analyze the preference of creams among subjects.

\section{RESULTS}

A total of 42 participants were enrolled (Fig. 1). The mean age of subjects was 11 years 5 months (8 years 1 month to 15 years 11 months). Twenty-four participants (57\%) were females. All participants completed the study.

The ceramide-based cream had a lower mean VAS score $(0.69, \quad \mathrm{SD}=1.63)$ for irritation 


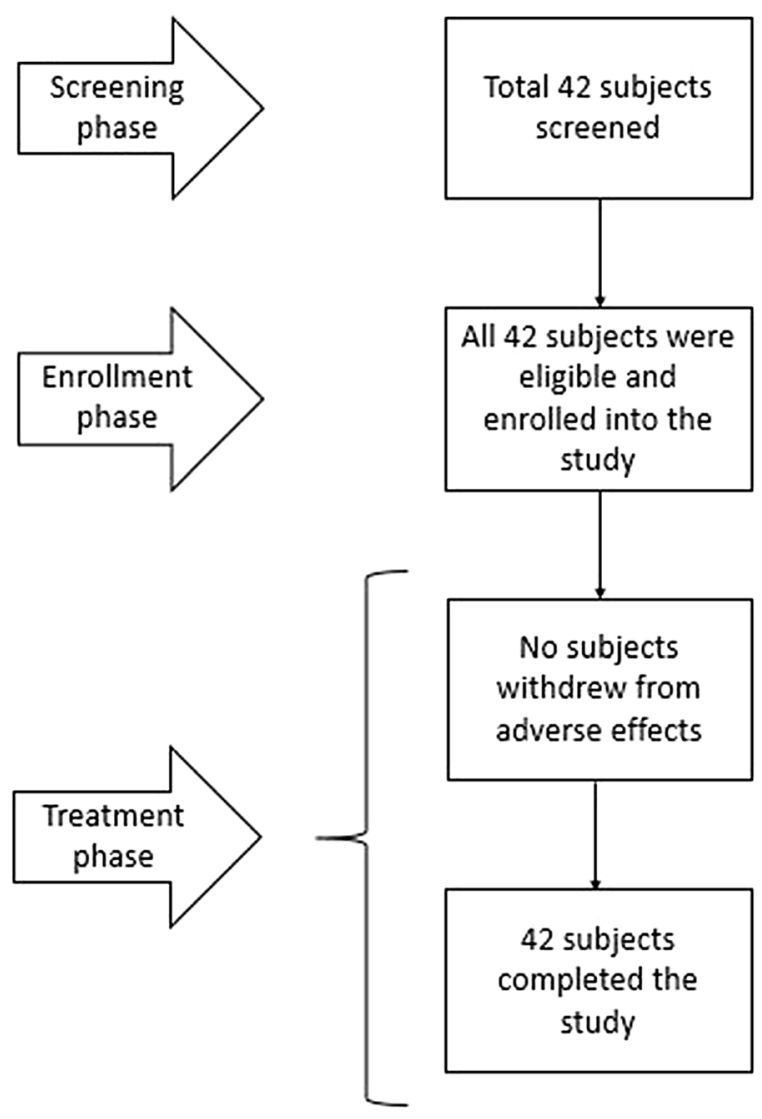

Fig. 1 Flow diagram of subject disposition

compared with the urea $5 \%$ cream $(1.43, \mathrm{SD}=$ 1.64). The range of responses of VAS scores for the two creams is shown in Fig. 2. Wilcoxon signed-rank test showed that this difference in irritation between the creams was significant, with the ceramide-based cream being less irritating than the urea $5 \%$ cream $(Z$-score -2.110 , $p=0.035$ ).

More participants also preferred the ceramide-based cream over the urea 5\% cream (62\% versus $38 \%$ ) as their daily moisturizer, but this did not reach statistical significance $(p=0.164)$.

\section{DISCUSSION}

Moisturizers are the mainstay of topical treatment of active $\mathrm{AD}$ flares as well as maintenance treatment to prevent flares [8]. However, compliance to frequent moisturization may be suboptimal due to various factors including pain and irritation when applied to affected skin, which can affect up to $27 \%$ of patients with $\mathrm{AD}$ [9]. Skin irritation is the most commonly reported adverse effect of moisturizers. This is usually more prominent on inflamed and broken skin but has been also reported on skin without obvious signs of inflammation or breakage. Other rarer adverse effects include irritant contact dermatitis, allergic contact dermatitis, occlusive folliculitis, photosensitive eruptions, acne cosmetica, and contact urticaria. Urea, lactic acid, glycerol, linoleic acid, and preservatives such as benzoic or sorbic acid have been reported to be associated with local irritation [6, 10-12]. Urea-containing creams, when used on $\mathrm{AD}$ patients, have been reported to produce stinging and burning sensations, itch, and even excoriations in some series, especially when used as a $10 \%$ preparation, with more acidic-based preparations or in combination with topical $1 \%$ hydrocortisone [11].

Our results suggest that this ceramide-based cream causes less irritation than the urea 5\% cream in $\mathrm{AD}$ children with subacute or acute flares, especially in the presence of skin excoriations. To date, this is one of the few studies to have directly compared the potential for irritation of two types of leave-on moisturizers in a pediatric population with $\mathrm{AD}$.

Parents and caregivers value their autonomy in the choice of moisturizers for their affected children and have different considerations in what they consider as ideal moisturizers. These include potential for skin irritation, vehicle thickness, greasiness, and cost. Often, they may be uncertain and even confused over the wide variety of commercial moisturizer products available. Rather than a trial-and-error approach, a more evidence-based approach is recommended [13]. The choice of moisturizers should also be suited to the patient's needs and preferences and based upon the skin condition, tolerability, climatic condition, lifestyle, and affordability [8, 14, 15]. Our findings support and add to the existing literature on the irritation potential of a commercially available moisturizer compared with urea cream in the treatment of $\mathrm{AD}$ and will be useful in guiding physicians on recommendations for 


\section{Frequency of irritability between Ceradan and Urea Cream}



Fig. 2 Visual analogue score (VAS) of irritation potential between Ceradan ${ }^{\circledR}$ and the urea cream

moisturizers for their AD patients as well as to improve patient compliance [10-12]. The urea cream can still be beneficial for $\mathrm{AD}$ children with nonexcoriated chronically xerotic skin for maintenance therapy but should be avoided in acute flares, especially in the presence of excoriations.

The main strength of our study is its randomized direct comparison of two different moisturizers concurrently in a single patient, minimizing confounding effects. In addition, skin irritation was objectively assessed using a validated severity scoring system. We acknowledge that staff and study personnel were not blinded, which may result in research bias, but due to the objective nature of the assessment and the lack of any further intervention from medical staff after the assessment was reported, this bias would have minimal effect on the study outcome.

Moisturizers should also be applied regularly over the body every day in $\mathrm{AD}$ patients and not just to a limited area that is in an acute flare. Our study was limited in the assessment of potential for irritation of the moisturizers in other areas of the body or after a regular consistent application over a longer duration. However, we believe that the antecubital fossae are common sites of flares in this age group and would be a good representative location. Furthermore, a single first-time test application in this age group of patients would likely suffice to influence their choice for their preferred moisturizer, especially if a noxious sensation were experienced.

Despite the relatively small sample size of our study, we were able to demonstrate a statistically significant lower VAS score for the ceramide-based cream compared with the urea $5 \%$ one. We believe that, with a larger sample size, the patient's preference for which moisturizer they would choose to use daily would have also reached statistical significance. In addition, assessment of compliance over a longer duration may have increased the strength of our study.

We recommend that future studies should include direct comparisons of not only the potential for irritation of a variety of eczemafriendly moisturizers and washes but also the greasiness and other common adverse effects and compliance over a longer period of use.

\section{CONCLUSIONS}

It is important to consider the potential for irritation of prescribed moisturizers in $\mathrm{AD}$ patients to improve compliance with treatment. 
Given the findings of our study, a ceramidebased moisturizer such as Ceradan ${ }^{\circledR}$ cream may be considered as a suitable choice for children to minimize irritation from moisturizer treatment of $\mathrm{AD}$, especially in acute flares.

\section{ACKNOWLEDGEMENTS}

Funding. The study and the journal's Rapid Service Fee were funded by Hyphens Pharma Pte Ltd, Singapore. Hyphens Pharma markets and sells Ceradan ${ }^{\circledR}$ cream, the ceramide-based moisturizer used in this study. ICA Pharma Pte Ltd markets and sells Aqurea Lite Cream, the urea 5\% moisturizer used in this study. The authors were responsible for the study design, data collection, data analysis, interpretation of results, manuscript preparation, and the decision to submit the manuscript for publication.

Authorship. All named authors meet the International Committee of Medical Journal Editors (ICMJE) criteria for authorship for this article, take responsibility for the integrity of this collective work, and have given their approval for this version to be published. The authors would like to thank the hospital staff who managed the clinical study and patients who participated in the study.

Disclosures. Valerie Pui Yoong Ho, Eric Ma, Hui Min Liew, Michelle Si Ying Ng, and Mark Jean Aan Koh have nothing to disclose.

Compliance with Ethics Guidelines. This study was approved by the SingHealth Centralised Institutional Review Board (CIRB), reference number 2015/3026, and conducted according to Good Clinical Practice guidelines and applicable local regulatory requirements. All procedures followed were in accordance with the ethical standards of the Helsinki Declaration of 1964, as revised in 2013. Written consent was obtained from parents or legal guardians, and written assent was obtained from patients.
Data Availability. The data supporting the conclusions are included within the manuscript.

Open Access. This article is licensed under a Creative Commons Attribution-NonCommercial 4.0 International License, which permits any non-commercial use, sharing, adaptation, distribution and reproduction in any medium or format, as long as you give appropriate credit to the original author(s) and the source, provide a link to the Creative Commons licence, and indicate if changes were made. The images or other third party material in this article are included in the article's Creative Commons licence, unless indicated otherwise in a credit line to the material. If material is not included in the article's Creative Commons licence and your intended use is not permitted by statutory regulation or exceeds the permitted use, you will need to obtain permission directly from the copyright holder. To view a copy of this licence, visit http://creativecommons.org/licenses/by$\mathrm{nc} / 4.0 /$.

\section{REFERENCES}

1. Cheok S, Yee F, Song Ma JY, et al. Prevalence and descriptive epidemiology of atopic dermatitis and its impact on quality of life in Singapore. Br J Dermatol. 2018;178:276-7.

2. Wollenberg A, Barbarot S, Bieber T, et al. Consensus-based European guidelines for treatment of atopic eczema (atopic dermatitis) in adults and children: part I. J Eur Acad Dermatol Venereol. 2018;32:657-82.

3. Moncrieff G, Cork M, Lawton S, Kokiet S, Daly C, Clark C. Use of emollients in dry-skin conditions: consensus statement. Clin Exp Dermatol. 2013;38: 231-8.

4. Chamlin SL, Kao J, Frieden IJ, et al. Ceramidedominant barrier repair lipids alleviate childhood atopic dermatitis: changes in barrier function provide a sensitive indicator of disease activity. J Am Acad Dermatol. 2002;47:198-208.

5. Bissonnette R, Maari C, Provost N, et al. A doubleblind study of tolerance and efficacy of a new ureacontaining moisturizer in patients with atopic dermatitis. J Cosmet Dermatol. 2010;9:16-211. 
6. Purnamawati S, Indrastuti N, Danarti R, Saefudin T. The role of moisturizers in addressing various kinds of dermatitis: a review. Clin Med Res. 2017;15: 75-877.

7. Draelos ZD. Differences in cutaneous irritation of five commonly used topical products. J Drugs Dermatol. 2016;15:870-3.

8. Van Zuuren EJ, Fedorowicz Z, Arents BWM. Emollients and moisturizers for eczema: abridged cochrane systematic review including GRADE assessments. Br J Dermatol. 2017;177:1256-71.

9. Huynh RK, Wong HH, Aw DCW, Toh MPHS. Adherence to topical corticosteroids and moisturisers in adults with endogenous eczema in Singapore. Hong Kong J Dermatol Venereol. 2015;23: 161-74.

10. Nasrollahi SA, Ayatollahi A, Yazdanparast T, et al. Comparison of linoleic acid-containing water-in-oil emulsion with urea-containing water-in-oil emulsion in the treatment of atopic dermatitis: a randomized clinical trial. Clin Cosmet Investig Dermatol. 2018;11:21-8.
11. Pan M, Heinecke G, Bernardo S, Tsui C, Levitt J. Urea: a comprehensive review of the clinical literature. Dermatol Online J. 2013;19:20392.

12. Lodén M, Andersson AC, Anderson C, et al. A double-blind study comparing the effect of glycerin and urea on dry, eczematous skin in atopic patients. Acta Derm Venereol. 2002;82:45-7.

13. Santer M, Muller I, Yardley L, Lewis-Jones S, Ersser S, Little P. Parents' and carers' views about emollients for childhood eczema: qualitative interview study. BMJ Open. 2016;6:e011887.

14. Sathishkumar D, Moss C. Topical therapy in atopic dermatitis in children. Indian J Dermatol. 2016;61: 656-61.

15. National Institute for Health and Care Excellence (NICE). Atopic eczema in under 12s: diagnosis and management. Published: 12 December 2007. www. nice.org.uk/guidance/cg57. Accessed Mar, 2020. 